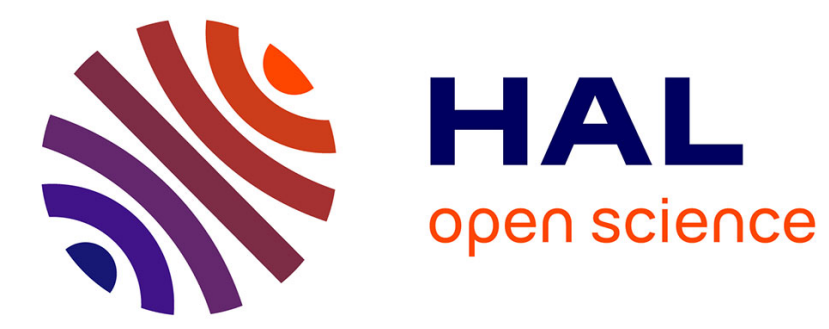

\title{
Pseudopower expansion of solutions of generalized equations and constrained optimization problems
}

\author{
J. Frederic Bonnans
}

\section{To cite this version:}

J. Frederic Bonnans. Pseudopower expansion of solutions of generalized equations and constrained optimization problems. [Research Report] RR-1956, INRIA. 1993. inria-00074717

\section{HAL Id: inria-00074717 \\ https://hal.inria.fr/inria-00074717}

Submitted on 24 May 2006

HAL is a multi-disciplinary open access archive for the deposit and dissemination of scientific research documents, whether they are published or not. The documents may come from teaching and research institutions in France or abroad, or from public or private research centers.
L'archive ouverte pluridisciplinaire HAL, est destinée au dépôt et à la diffusion de documents scientifiques de niveau recherche, publiés ou non, émanant des établissements d'enseignement et de recherche français ou étrangers, des laboratoires publics ou privés. 


\section{Pseudopower Expansion of Solutions of Generalized Equations and Constrained Optimization Problems}

Joseph-Frédéric BONNANS

Agnès SULEM

$N^{\circ} 1956$

Juillet 1993

PROGRAMME 5

Traitement du signal, automatique et productique

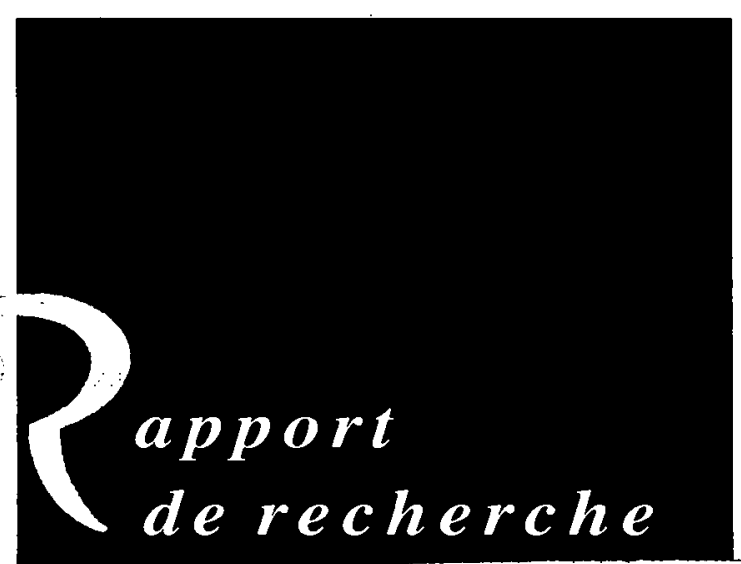




\title{
Pseudopower expansion of solutions of generalized equations and constrained optimization problems
}

\author{
Pseudo-développement de solutions d'équations \\ généralisées et de problèmes d'optimisation avec \\ contraintes
}

\author{
Joseph Frédéric Bonnans* Agnès Sulem*
}

July 13, 1993

\begin{abstract}
We compute the solution of a strongly regular perturbed generalized equation as the sum of a pseudopower expansion, i.e. the expansion at order $k$ is the solution of the generalized equation expanded at order $k$ and thus depends itself on the perturbation parameter $\varepsilon$. In the polyhedral case, the pseudopower expansion reduces to a classical Taylor expansion. For constrained optimization problems with strongly regular solution, we check that the quadratic growth condition holds and that, at least locally, solutions of the problem and solutions of the associated optimality system coincide. In the special case of a finite number of inequality constraints, the solution and the Lagrange multiplier can be expanded in Taylor series if the gradients of the active constraints are linearly indepcndent. If the data are analytic, the solution and the multiplier are analytic functions in $\varepsilon$ provided that some strong second order condition holds.
\end{abstract}

Résumé : On démontre que la solution d'une équation généralisée fortement régulière perturbée par un paramètre $\approx$ se développe en puissances de la perturbation, le développement d'ordre $k$ étant solution de l'équation généralisée développée à l'ordre $k$. Dans le cas polyédral, ce pseudo-développement se réduit à un développement de Taylor classique. Cés résultats sont appliqués au cas de problèmes de perturbations régulières en optimisation sous contraintes. On vérifie que, sous l'hypothèse de forte régularité, la propriété de croissance quadratique est satisfaite, et les solutions du problème d'optimisation et du système d'optimalité correspondant coïncident localement. Dans le cas standard de la programmation non linéaire (nombre fini de contraintes d'inégalité) on démontre, sous l'hypothèse d'indépendance linéaire des gradients des

*INRIA, Domaine de Voluceau, BP 105, 78153 Rocquencourt, France. 
contraintes actives, que la solution du problème d'optimisation et le multiplicateur de Lagrange associé se développent en série de Taylor. Dans le cas de clonnées analytiques et sous une hypothèse du deuxième ordre, la solution et le multiplicateur sont des fonctions analytiques de $\varepsilon$.

Key Words : generalized equations, variational inequalities, nonlinear programming, sensitivity analysis, powerseries, strong regularity, constrained optimization, perturbation theory.

\section{Introduction}

In this paper we deal with a technique that we call pseudopower expansion, for which we refer to A. Bensoussan [1] . We expose the idea in the simple case of a minimization problem

$$
\min f(x, \varepsilon) ; x \in \kappa
$$

where $X$ is a Banach space, $f$ is a smooth function from $X^{-} \times \mathbb{P}^{+}$to $\mathbb{P}$, and $K$ is a closed convex subset of $X$. Assuming that $(P)_{0}$ has a unique solution $x^{0}$, we compute a solution $x^{\varepsilon}$ of the perturbed problem $(P)_{\varepsilon}$, of the form :

$$
x^{\varepsilon}=x^{0}+\varepsilon x^{1}(\varepsilon)+\varepsilon^{2} x^{2}(\xi)+\cdots
$$

that is, the coefficients of the powers of $\varepsilon$ depend themself of $\varepsilon$ (this motivates the terminology of pseudopower expansion). The optimality system of $(P)=$ can be written as

$$
\nabla f\left(x^{\varepsilon}, \Xi\right)+N_{\kappa}\left(x^{\varepsilon}\right) \ni 0
$$

where $\nabla$ denotes the gradient with respect to $x, N_{K}(x)$ is the set of outward normals to $K$ at $x$, defined by

$$
N_{K}(x)= \begin{cases}\emptyset & \text { if } x \notin K^{\prime}, \\ \left\{w \in X^{\prime} ;\langle w, y-x\rangle \leq 0 . \quad \forall y \in K^{\prime}\right\} & \text { if } x \in K^{\prime},\end{cases}
$$

and $X^{\prime}$ is the topological dual of $\mathcal{X}$. The idea is to expand the generalized equation (1.2), using the a priori pseudopower expansion (1.1). At the first order. we obtain $x^{1}(\varepsilon)$ solution of

$$
\nabla f\left(x^{0}, 0\right)+\varepsilon \nabla f_{\varepsilon}^{\prime}\left(x^{0}, 0\right)+\varepsilon \Gamma^{2} f\left(x^{0}, 0\right) x^{1}(\varepsilon)+N_{K}\left(x^{0}+\varepsilon x^{1}(\varepsilon)\right) \ni 0
$$

where $\nabla^{2}$ is the Hessian with respect to $x$. Then. $x^{1}(\varepsilon)$ being fixed, we compute $x^{2}(\varepsilon)$ by expanding (1.2) at order 2, and so forth.

Note that first order expansions or pseudoexpansions of solutions of nonlinearly constrained optimization problems can be obtained under weak hypotheses (see e.g. Bonnans-Ioffe-Shapiro [4] for nonlinear programming problems. Bommans-Shapiro [5], Kunish and Ito [9], Malanowski [12] for optimization problems in Banach spaces, Robinson [16] and Shapiro [18] for generalized 
equations). Here we need strong stability hypotheses in order to compute the pseudopower expansion at any order.

The aim of the paper is twofold. First, we study this technique for an abstract generalized equation with a general closed multivalued operator. We justify the expansion at any order under the strong regularity (SR) condition (Robinson [15]) recalled below. We prove that the coefficients of the expansion are independent of $\varepsilon$, when $K$ is polyhedral in a sense made precise below, and when an hypothesis involving the linear independence of the gradient of active constraints holds. In this framework, assuming that the data are analytic, we obtain the powerseries expansion of the solutions under an hypothesis implying the strict complementarity condition for the perturbed problem only.

The second part of this paper is devoted to the application of these results to the perturbation theory for constrained optimization problems. We prove that, under the SR condition, the local solutions of the perturbed problem are well defined and coincide with the solutions of the associated optimality system which can be written as a generalized equation. Sufficient conditions for strong regularity are given. They are related to some results of Kunish and Ito [9] and Malanowski [12]. Each term of the expansion of the solution can be interpreted as the solution of an optimization problem with quadratic cost and linear constraints. In the special case of nonlinear programming, we prove that under the hypotheses of the theorem of Jittorntrum [10] (stating, in short, directional differentiability of a strongly regular solution satisfying a strong second order condition), the solution has indecd a power expansion (or powerseries) at any order, provided of course that the data have enough regularity. In the case of convex problems, we recover the result of Malanowshi [13]. In addition, if the data are analytic. then the solution is itself analytic in $\varepsilon$.

\section{Pseudopower expansion of solutions of strongly reg- ular generalized equations}

Let $Z$ and $E$ be two Banach spaces, and $F$ a mapping $Z \times \mathbb{R}^{+} \rightarrow E$. Let $z \rightarrow N(z)$ be a multivalued operator with closed graph from $Z$ to $E$; i.e. for each $z \in Z, N(z)$ is a (possibly: empty) subset of $E$ such that if $z^{n} \rightarrow z$ in $Z, e^{n} \rightarrow \epsilon$ in $E$, and $\epsilon^{n} \in N\left(z^{n}\right)$, then $\epsilon \in \Lambda(z)$. We consider the generalized equation

$$
F(z, \varepsilon)+N(z) \ni 0 .
$$

If $E=Z^{\prime}$, the topological dual of $Z$, and $N(z)$ is the set of outward nomals $N_{K}(z)$ to a given closed convex cone $K$ in $Z$, we recover the classical definition of a generalized equation (see Robinson [15]). However, in order to embedd in our framewort the constrained optimization problem of section 4, we need this more general format which does not add any essentiel difficulty (see Remark 4.1).

Assuming that $z^{0}$ is a solution of $(V I)_{0}$, we compute an expansion of the solution $z^{\varepsilon}$ of $(V I)_{\varepsilon}$ such that

$$
z^{\varepsilon}=z^{u}+\varepsilon z^{1}(\varepsilon)+\varepsilon^{2} z^{2}(\varepsilon)+\cdots
$$


where $z^{k}(\varepsilon), k \geq 1$ are solutions of "linear" generalized equations of the form

$$
A^{k} z+N(z) \ni b^{k}
$$

with $A^{k} \in L(Z, E)$ and $b^{k} \in E$. For this purpose, we assume $F$ to be (for simplicity) $C^{\infty}$ and we use the concept of strong regularity, due to Robinson [15].

Definition 2.1 We say that $z^{0}$ is a strongly regular (SR) solution of $(V I)_{0}$ if there exists a neighborhood $\mathcal{V} \times \mathcal{O}$ of $\left(z^{0}, 0\right)$ in $Z \times E$ and $\beta>0$ such that the linear generalized equation

$$
F\left(z^{0}, 0\right)+F_{z}^{\prime}\left(z^{0}, 0\right)\left(z-z^{0}\right)+N(z) \ni \delta
$$

admits, for any $\delta$ in $\mathcal{O}$, a unique solution $z(\delta)$ in $\mathcal{V}$ such that

$$
\left\|z\left(\delta^{1}\right)-z\left(\delta^{2}\right)\right\| \leq \beta\left\|\delta^{2}-\delta^{1}\right\|, \text { for all } \delta^{1}, \delta^{2} \text { in } \mathcal{O} .
$$

Let us admit for the moment that the functions $z^{k}(\varepsilon)$ appearing in (2.1) are bounded (for $\varepsilon$ small enough), we may compute an expansion of $F\left(z^{\varepsilon}, \varepsilon\right)$ : for instance, at order 2 we get

$$
\begin{aligned}
F\left(z^{\varepsilon}, \varepsilon\right) & =F\left(z^{0}, 0\right)+\varepsilon\left[F_{\varepsilon}^{\prime}\left(z^{0}, 0\right)+F_{z}^{\prime}\left(z^{0}, 0\right) z^{1}(\varepsilon)\right] \\
& +\varepsilon^{2}\left[\frac{1}{2} F_{z^{2}}^{\prime \prime}\left(z^{0}, 0\right)\left(z^{1}(\varepsilon), z^{1}(\varepsilon)\right)+F_{z \varepsilon}^{\prime \prime}\left(z^{0}, 0\right) z^{1}(\varepsilon)+\frac{1}{2} F_{\varepsilon^{2}}^{\prime}\left(z^{0}, 0\right)\right] \\
& +\varepsilon^{2} F_{z}^{\prime}\left(z^{0}, 0\right) z^{2}(\varepsilon)+O\left(\varepsilon^{3}\right) .
\end{aligned}
$$

More generally, denote by $\Phi^{k}\left(z^{0}, \ldots, z^{k}(\varepsilon), \varepsilon\right)$ the expansion of $F\left(z^{\varepsilon}, \varepsilon\right)$ up to order $k$, we have the following inductive relation (see Bensoussan [1])

$$
\Phi^{0}\left(z^{0}, \varepsilon\right):=F\left(z^{0}, 0\right)
$$

and for $k \geq 1$

$$
\Phi^{k}\left(z^{0}, \ldots, z^{k}, \varepsilon\right):=\Phi^{k-1}\left(z^{0}, \ldots, z^{k-1}, \varepsilon\right)+\varepsilon^{k} \psi^{k}\left(z^{0}, \ldots, z^{k-1}\right)+\varepsilon^{k} F_{z}^{\prime}\left(z^{0}, 0\right) z^{k}
$$

where $\psi^{k}$ is defined by

$$
\psi^{1}\left(z^{0}\right)=F_{\varepsilon}^{\prime}\left(z^{0}, 0\right)
$$

and for $k>1$,

$$
\begin{aligned}
& \psi^{k}\left(z^{0}, \ldots, z^{k-1}\right)=\frac{1}{k !} F_{z^{k}}^{(k)}\left(z^{0}, 0\right)+\sum_{n=1}^{k-1} \frac{1}{n !} F_{z \varepsilon^{n}}^{(n+1)}\left(z^{0}, 0\right) z^{k-n} \\
& +\sum_{n=2}^{k} \sum_{j=0}^{k-n} \sum_{\substack{i_{1}+\cdots+i_{n}=k-j \\
i_{1}, \ldots, i_{n} \geq 1}} \frac{1}{n !} \frac{1}{j !} F_{z^{n} \varepsilon^{\prime}}^{(n+j)}\left(z^{0}, 0\right) z^{i_{1}} \ldots, z^{i^{n}} .
\end{aligned}
$$

We obtain for instance

$$
\Phi^{1}\left(z^{0}, z^{1}, \varepsilon\right):=F\left(z^{0}, 0\right)+\varepsilon\left[F_{\varepsilon}^{\prime}\left(z^{0}, 0\right)+F_{z}^{\prime \prime}\left(z^{0}, 0\right) z^{1}\right] .
$$


Suppose that $z^{1}(\varepsilon), \ldots, z^{k-1}(\varepsilon)$ are uniquely determined. We can define by induction the function $z^{k}(\varepsilon)$ as the solution of the linear generalized equation (expansion at order $k$ of $(V I)_{\varepsilon}$ )

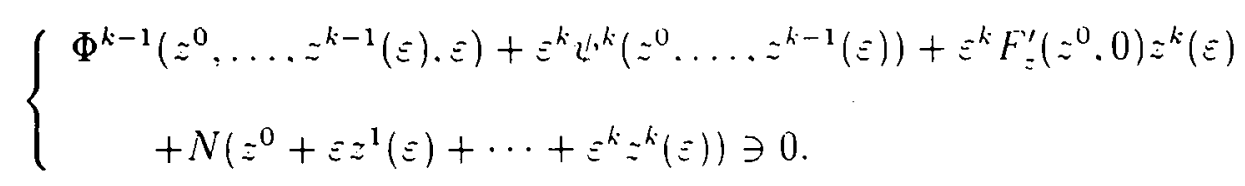

The main result of this section is:

Theorem 2.1 Assume that $\tilde{z}^{0}$ is a strongly regular solution of $(V I)_{0}$. Then there exists $\varepsilon^{0}>0$ and a neighborhood $\mathcal{V}$ of $z^{0}$ in $Z$ such that for all $\varepsilon$ in $\left[0, \varepsilon^{0}\right]$ :

(i) $(V I)_{\varepsilon}$ has a unique solution $z^{\varepsilon}$ in $\mathcal{V}$, and $z^{\varepsilon}=z^{0}+O(\varepsilon)$.

(ii) Formula (2.5) defines for all ki a bounded mapping $:^{*}(\Sigma):\left(0 . \Sigma_{0}\right] \rightarrow \mathcal{V}$,

(iii) For all $k$ we have the expansion

$$
\tilde{z}^{\varepsilon}=z^{0}+z^{1}(\xi)+\cdots+\varepsilon^{k} z^{k}(\varepsilon)+O\left(\varepsilon^{k+1}\right) .
$$

\section{Proof}

(i) This is essentially the result of Robinson ([15]. Theorem 2.1), although we state it for a general closed operator $N$ in the Banach space $E$ : this however does not change the proof, that henceforth is not repeated.

(ii) We use an induction argument. The result is true at step $k=0$ as $z^{0}$ is constant. Assume now that $z^{0}$ to $z^{k-1}$ (we omit the argument $z$ for simplicity) are bounded and set

$$
z^{k}:=z^{0}+\cdots+z^{k} z^{k} \text {. }
$$

Write $(2.5)$ as

$$
\begin{aligned}
F\left(z^{0}, 0\right)+F_{z}^{\prime}\left(z^{0}, 0\right)\left(z^{k}-z^{0}\right)+N\left(z^{k}\right) & \ni F\left(z^{0} \cdot 0\right)-\Phi^{k-1}\left(z^{0} \ldots, z^{k-1}, \varepsilon\right)-\varepsilon^{k} \psi^{k}\left(z^{0}, \ldots, z^{k-1}\right) \\
& +F_{z}^{\prime}\left(z^{0} \cdot 0\right)\left(z^{k-1}-z^{0}\right),
\end{aligned}
$$

and compare with (2.5) at order $k-1$, which can be written as

$$
\begin{aligned}
F\left(z^{0}, 0\right)+F_{z}^{\prime}\left(z^{0}, 0\right)\left(z^{k-1}-z^{0}\right)+N\left(z^{k-1}\right) & \ni F\left(z^{0}, 0\right)-\Phi^{k-1}\left(z^{0}, \ldots, z^{k-1}, \varepsilon\right) \\
& +F_{z}^{\prime}\left(z^{0}, 0\right)\left(z^{k-1}-z^{0}\right) .
\end{aligned}
$$

Using the strong regularity of $z_{0}$ and the fact that the right hand sides of (2.7) and (2.8) go to 0 when $\varepsilon$ tends to 0 and differ by order $O\left(\varepsilon^{k}\right)$. we get the existence of $z^{k}$ in a neighborhood of $z^{0}$, and of $z^{k}$ such that

$$
\varepsilon^{k} z^{k}=z^{k}-z^{k-1}=O\left(z^{k} i^{k}\left(z^{0} \ldots \ldots z^{k-1}\right)\right)=O\left(z^{k}\right)
$$


which implies that $z^{k}$ is bounded.

(iii) Again we proceed by induction. Equation (2.6) is satisfied for $k=0$ from point (i). Define $r^{k}$ by

$$
z^{\varepsilon}=z^{0}+\cdots+\varepsilon^{k-1} z^{k-1}+\varepsilon^{k} r^{k} .
$$

Assuming (2.6) to hold at step $k-1$ (this is true for $k=1$ ), we deduce that $r^{k}=O(1)$. Expanding $F\left(z^{\varepsilon}, \varepsilon\right)$ at order $k$, we get

$$
F\left(z^{\varepsilon}, \varepsilon\right)=\Phi^{k}\left(z^{0}, \ldots, z^{k-1}, r^{k}, \varepsilon\right)+O\left(\varepsilon^{k+1}\right),
$$

hence

$$
\Phi^{k}\left(z^{0}, \ldots, z^{k-1}, r^{k}, \varepsilon\right)+N\left(z^{\varepsilon}\right) \ni O\left(\varepsilon^{k+1}\right)
$$

that is, using $(2.2)$ :

$$
\begin{aligned}
F\left(z^{0}, 0\right)+F_{z}^{\prime}\left(z^{0}, 0\right)\left(z^{\varepsilon}-z^{0}\right)+N\left(z^{\varepsilon}\right) & \ni F\left(z^{0}, 0\right)-\Phi^{k-1}\left(z^{0}, \ldots \ldots z^{k-1}, \varepsilon\right)-z^{k} \psi^{k}\left(z^{0}, \ldots, z^{k-1}\right) \\
& +F_{z}^{\prime}\left(z^{0} \cdot 0\right)\left(z^{k-1}-z^{0}\right)+O\left(z^{k+1}\right) .
\end{aligned}
$$

Comparing to (2.7) and using SR, we get $z^{z}=z^{k}+O\left(z^{k+1}\right)$. which is equivalent to (2.6) at order $k$.

Remark 2.1 The pseudopower expansion can be used in order to compute a numerical approximation of $z^{\varepsilon}$. It is thus important to evaluate the precision required for the computation of each term of the expansion. It can be checked that the propagation of errors is such that in order to obtain $z^{k}$ with an error in $o\left(\xi^{k}\right)$, the computation of $z^{i}$ for $i=1, \ldots, k-1$ is required with an error in $o\left(\varepsilon^{k-i}\right)$. Indeed, using $S R$ and (2.5). we set that it is sufficient to evaluate $\Phi^{k-1}\left(z^{0}, \ldots, z^{k-1}, \varepsilon\right)$ and $\varepsilon^{k} \psi^{k}\left(z^{0}, \ldots, z^{k-1}(\xi)\right)$ with an error in o( $\left.z^{k}\right)$. From the relation

$$
\Phi^{k-1}\left(z^{0}, \ldots, z^{k-1}, \Xi\right)=F\left(z^{0}, 0\right)-F_{z}^{\prime}\left(z^{0}, 0\right) z^{0}+\sum_{i=1}^{k-1} z^{i} z^{\prime i}\left(z^{0} \ldots, z^{i-1}\right)+F_{z}^{\prime}\left(z^{0}, 0\right) z^{k-1}
$$

we deduce that this is achieved when, on the one hand. each $i^{i}\left(z^{0}, \ldots, z^{i-1}\right)$ for $i=1$ to $k-1$ is computed with an error in o( $\left.\varepsilon^{k-i}\right)$ and on the other hand. $:^{0} \ldots \ldots z^{k-1}$ are computed with an error in o(1). In particular. this is the case if cach $z^{i}$ is computed with an error in $o\left(\varepsilon^{k-i}\right)$.

\section{The polyhedral case : classical series and power ex- pansion of solutions}

In this section we set a framework in which the coefficients $z^{k}(\Sigma)$ of the expansion of $z^{2}$ given in (2.6) actually do not depend on $\Xi$ for $\Xi$ small enough. Roughly speaking, this is obtained when $K$ is defined by linear equalities and a finite number of linear inequalities, with a condition of linear independence of the gradients of the active constraints. 


\subsection{Finite number of nonnegativity constraints}

We start with the following case, corresponding to a finite number of nonnegativity constraints.

$$
\begin{aligned}
Z & =\mathcal{X} \times \mathbb{P}^{p}, \\
\tilde{z} & =(x, y), \\
E & =E_{1} \times \mathbb{P}^{p}, \\
N(x, y) & :=\left(\begin{array}{c}
0 \\
N_{\mathbb{R}^{p+}}(y)
\end{array}\right)
\end{aligned}
$$

with $X$ and $E_{1}$ two Banach spaces. Then, (VI) $)_{\varepsilon}$ is equivalent to

$$
\left\{\begin{array}{l}
F(x, y, \varepsilon)+\left(\begin{array}{l}
0 \\
\mu
\end{array}\right)=0, \\
\mu \geq 0, y \geq 0, y^{t} \mu=0 .
\end{array}\right.
$$

Proceeding as in section 2, we assume that $\left(x^{0}, y^{0}\right)$ is a strongly regular solution of $(V I)_{0}$ and we consider the expansion at order $k$ of $(3.1)$ :

$$
\left\{\begin{array}{l}
\Phi^{k-1}\left(z^{0}, \ldots, z^{k-1}(\varepsilon), \varepsilon\right)+\varepsilon^{k} \psi^{k}\left(z^{0}, \ldots, z^{k-1}(\varepsilon)\right)+\varepsilon^{k} F_{z}^{\prime}\left(z^{0}, 0\right) z^{k}(\varepsilon) \\
\quad+\left(\begin{array}{c}
0 \\
\tilde{\mu}^{k}(\varepsilon)
\end{array}\right)=0, \\
\tilde{z}^{k}:=z^{0}+\varepsilon z^{1}(\varepsilon)+\cdots+\varepsilon^{k} z^{k}(\varepsilon) . \\
z^{k}(\varepsilon)=\left(x^{k}(\varepsilon), y^{k}(\varepsilon)\right), \\
\tilde{y}^{k}(\varepsilon) \geq 0, \tilde{\mu}^{k}(\varepsilon) \geq 0,\left(\tilde{y}^{k}(\varepsilon)\right)^{t} \tilde{\mu}^{k}(\varepsilon)=0 .
\end{array}\right.
$$

We set for $k \geq 1$

$$
\mu^{k}(\varepsilon):=\left(\tilde{\mu}^{k}(\varepsilon)-\tilde{\mu}^{k-1}(\varepsilon)\right) / \varepsilon^{k} .
$$

It follows that

$$
\mu^{k}(\varepsilon)=\mu^{0}+\varepsilon \mu^{1}(\varepsilon)+\cdots+\varepsilon^{k} \mu^{k}(\varepsilon) .
$$

Define the index sets

$$
\begin{aligned}
& I_{+}^{0}:=\left\{i \in\{1, \ldots, p\} ; y_{i}^{0}=0: \mu_{i}^{0}>0\right\}, \\
& I_{0}^{0}:=\left\{i \in\{1, \ldots, p\} ; y_{i}^{0}=0: \mu_{i}^{0}=0\right\}, \\
& I_{-}^{0}:=\left\{i \in\{1, \ldots, p\} ; y_{i}^{0}>0\right\},
\end{aligned}
$$

and assume that $\left(\varepsilon^{\prime}(\varepsilon), \mu^{\prime}(\varepsilon)\right)$ do not depend on $\varepsilon$ for $c=0, \ldots k-1$ when $\varepsilon<\varepsilon^{0}$ : this is at least the case when $k=1$. We define by induction for $r \geq 1$ the system 


$$
\left\{\begin{array}{l}
F_{z}^{\prime}\left(z^{0}, 0\right) z^{k}+\psi^{k \cdot k}\left(z^{0}, \ldots, z^{k-1}\right)+\left(\begin{array}{c}
0 \\
\mu^{k}
\end{array}\right)=0, \\
y_{i}^{k}=0, i \in I_{+}^{k-1}, \\
y_{i}^{k} \geq 0, \mu_{i}^{k} \geq 0, i \in I_{0}^{k-1}, \\
\mu_{i}^{k}=0, i \in I_{-}^{k-1}, \\
\left(\mu^{k}\right)^{t} y^{k}=0
\end{array}\right.
$$

and the set of indices

$$
\begin{aligned}
& I_{+}^{\ell}:=I_{+}^{\ell-1} \cup\left\{i \in I_{0}^{\ell-1} ; \mu_{i}^{\ell}>0\right\}, \\
& I_{0}^{\ell}:=\left\{i \in I_{0}^{\ell-1} ; y_{i}^{\ell}=\mu_{i}^{\ell}=0\right\}, \\
& I_{-}^{\ell}:=I_{-}^{\ell-1} \cup\left\{i \in I_{0}^{\ell-1} ; y_{i}^{\ell}>0\right\} .
\end{aligned}
$$

Note that $I_{+}^{k}$ and $I_{-}^{k}$ are monotonously increasing sequences, and that $I_{0}^{k}$ is monotonously decreasing; hence, for $k$ large enough, they are identical to some fixed sets $\left(I_{+}, I_{-}, I_{0}\right)$.

Theorem 3.1 Assume that $z^{0}$ is a strongly regular solution of $(V I)_{0}$ and $K$ is polyhedral. Then

(i) For all $k \geq 0$, there exists $\equiv \geq 0$ such that the functions $z^{\ell}(\varepsilon)$ solution of (3.2), $\ell \leq k$ do not depend on $\varepsilon$ when $\varepsilon<\bar{\varepsilon}$, i.e. there exist $z^{1}, \ldots, z^{k}$ in $Z$ such that

$$
z^{\varepsilon}=z^{0}+\varepsilon z^{1}+\cdots+\varepsilon^{k} z^{k}+O\left(\varepsilon^{k+1}\right),
$$

and a similar expansion holds for the multiplier, i.e.

$$
\mu^{\varepsilon}=\mu^{0}+\varepsilon \mu^{1}+\cdots+\varepsilon^{k} \mu^{k}+O\left(\varepsilon^{k+1}\right) .
$$

At each step $k,\left(z^{k}, \mu^{k}\right)$ is the unique solution of (3.3).

(ii) For any $I$ such that.

$$
I_{+} \subset I \subset I_{+} \cup I_{0}
$$

the Taylor expansions of $\left(z^{*}, \mu^{\varepsilon}\right)$ do satisfy the equations of the Taylor expansion of the nonlinear equation

$$
\left\{\begin{array}{l}
F\left(z^{\varepsilon}, \varepsilon\right)+\left(\begin{array}{c}
0 \\
\mu^{\varepsilon}
\end{array}\right)=0, \\
y_{i}^{\varepsilon}=0, i \in I ; \mu_{i}^{\varepsilon}=0, i \notin I .
\end{array}\right.
$$


Proof. (i) We proceed by induction. From (3.1) with $z=0$ we have

$$
\left\{\begin{array}{l}
F\left(z^{0}, 0\right)+\left(\begin{array}{c}
0 \\
\mu^{0}
\end{array}\right)=0 . \\
y_{i}^{0} \geq 0, \mu_{i}^{0} \geq 0, i \in I\left(z^{0}\right) ; \mu_{i}^{0}=0, i \notin I\left(z^{0}\right) .
\end{array}\right.
$$

where

$$
I\left(z^{0}\right):=\left\{i \in\{1 \ldots, p\}: y_{i}^{0}=0\right\}
$$

Expansion of (3.1) at order one gives

$$
\left\{\begin{array}{l}
F\left(z^{0} \cdot 0\right)+\Sigma\left[F_{z}^{\prime}\left(z^{0} \cdot 0\right) z^{1}(\Xi)+F_{s}^{\prime}\left(z^{0} \cdot 0\right)\right]+\left(\begin{array}{c}
0 \\
\mu^{0}+\Sigma \mu^{1}(\xi)
\end{array}\right)=0, \\
y^{0}+\Sigma y^{1}(\Sigma) \geq 0 . \quad \mu^{0}+\Sigma \mu^{1}(\Sigma) \geq 0 . \\
\left(\mu^{0}+\varepsilon \mu^{1}(\varepsilon)\right)^{t}\left(y^{0}+\varepsilon z^{1}(\Sigma)\right)=0 .
\end{array}\right.
$$

As $z^{1}(\varepsilon)$ is bounded by Theorem 2.1. the inequality constraints which are not in $I\left(z^{0}\right)$ are not active for (3.7) for $\varepsilon$ smaller than some $\varepsilon_{1}>0$. Using now (3.7) and the property that $\mu^{1}(\varepsilon)$ is bounded, we infer that $\mu^{1}(\varepsilon):=\mu^{0}+\varepsilon \mu^{1}(\varepsilon)$ is bounded and converges to $\mu^{0}$. It follows from (3.7) that $y_{i}^{1}(\varepsilon)=0$ whenever $i$ is in $I_{+}^{0}$ and $z$ is small enough, say $\varepsilon<\varepsilon_{2}$. Using (3.7), we deduce that when $\varepsilon<\min \left(\varepsilon_{1} . \varepsilon_{2}\right)$, the couple $\left(z^{1}(\Sigma) \cdot \mu^{1}(\Sigma)\right)$ is a solution of $(3.7)$ iff it satisfies

$$
\left\{\begin{array}{l}
F_{z}^{\prime}\left(z^{0}, 0\right) z^{1}(\varepsilon)+F_{s}^{\prime}\left(z^{0}, 0\right)+\left(\begin{array}{c}
0 \\
\mu^{1}(\varepsilon)
\end{array}\right)=0, \\
y_{i}^{1}(\varepsilon)=0, i \in I_{+}^{0}, \\
y_{i}^{1}(\varepsilon) \geq 0, \mu_{i}^{1}(\varepsilon) \geq 0 . \mu_{i}^{1}(\xi) y_{i}^{1}(\varepsilon)=0, i \in I_{0}^{0}: \\
\mu_{i}^{1}(\varepsilon)=0, i \in I_{-}^{0} .
\end{array}\right.
$$

Since (3.7) has a unique solution for any $z$. and (3.8) is a system of inequalities whose clata do not depend on $\varepsilon$. it follows that $(3.8)$ has a unique solution $\left(z^{1}, \mu^{1}\right)$ independent of $\varepsilon$.

Proceeding in a similar way at step $k$. we check that $\left(z^{k}, \mu^{k}\right)$ is a solution of $(3.2)$ iff $\left(z^{k}, \mu^{k}\right)$ is a solution of $(3.3)$.

(ii) It follows from point (i) that

- If $i \in I_{+}$, then $\mu_{i}^{*}$ is positive whenever $\varepsilon$ is positive and small enough, and the constraint $y_{i}^{\varepsilon} \leq 0$ is active. i.e. $y_{i}^{\varepsilon}=0, \forall i \in I_{+}$:

- If $i \in I_{-}$, then $y_{i}^{\varepsilon}>0$, i.e. the constraint is not active for $\varepsilon$ positive small enough. 
Now the coefficient at order $k$ of the expansion of the solution of (3.5) satisfies :

$$
\left\{\begin{array}{l}
F_{z}^{\prime}\left(z^{0}, 0\right) z^{k}+v^{k}\left(z^{0}, \ldots, z^{k-1}\right)+\left(\begin{array}{c}
0 \\
\mu^{k}
\end{array}\right)=0, \\
\left(y^{k}\right)_{I}=0, \mu_{i}^{k}=0 \text { if } i \notin I .
\end{array}\right.
$$

It follows from the definition of $I$ that the solution $\left(z^{k}, \mu^{k}\right)$ of $(3.3)$ is a solution of (3.9), as was claimed.

Remark 3.1 Let us define

$$
C_{k}:=\left\{y \in \mathbb{F}^{p} ; y_{i}=0, i \in I_{+}^{k-1}: y_{i} \leq 0 . i \in I_{0}^{k-1}\right\}
$$

and

$$
N_{k}(x, y)=\left(\begin{array}{c}
0 \\
N_{C_{k}}(y)
\end{array}\right)
$$

It appears that the system (3.3) defining $\left(\tilde{z}^{k}, \mu^{k}\right)$ is equicalent to the generalized equation

$$
F_{z}^{\prime}\left(z^{0}, 0\right) z^{k}+\psi^{k}\left(z^{0}, \ldots z^{k-1}\right)+N_{k}\left(z^{k}\right) \ni 0 .
$$

Similarly, for I satisfying (3.4), define

$$
C:=\left\{y \in \mathbb{Z}^{p}: y_{i}=0 . i \in I\right\} .
$$

We may observe that (3.5) is equivalent to

$$
F\left(z^{\Sigma}, \Xi\right)+V\left(z^{\approx}\right) \ni 0
$$

with $N(x, y)=\left(\begin{array}{c}0 \\ N_{C}(y)\end{array}\right)$

In Theorem 3.1, we noticed that the expansion of the solution of the generalized equation is also an expansion of the solution of the system of equalities (3.5). However the uniqueness of the expansion of (3.5) is not garanteed. since the solution of (3.9) is not necessarily unique. A sufficient condition for uniqueness is given below.

Proposition 3.1 Assume that $I_{0}=0$ and that the hypotheses of Theorem 3.1 are satisfied. Then:

(i) for each $k \geq 1$. for fixed $\left(z^{1} \ldots \ldots z^{k-1}\right)$. equation (3.9) has a unique solution $\left(z^{k}, \mu^{k}\right)$,

(ii) if in addition $\mathrm{X}=\mathbb{X}^{q}$ and $F$ is analytic. then the function $\Xi \rightarrow z^{2}$ is itself analytic. 
Proof Let $\hat{k}$ be such that $I_{u}^{\hat{k}-1}=\emptyset$. Consider the perturbed generalized equation

$$
F(z, \varepsilon)+\frac{\varepsilon^{\dot{k}}}{\hat{k} !} \delta+N(z) \ni 0
$$

with $\delta \in Z^{\prime}$ given, and denote the solution by $z_{\tilde{\delta}}^{\varepsilon}$. For $\varepsilon=0$, (3.10) has the same strongly regular solution $z^{0}$ as $(3.1)$. It is clear that its pseudoexpansion $\left\{\tilde{z}_{s}^{k}\right\}$ satisfies

$$
z^{k}(\varepsilon)=z_{\delta}^{k}(\varepsilon), \quad k<\hat{k},
$$

and similarly

$$
\mu^{k}(\varepsilon)=\mu_{\delta}^{k}(\varepsilon), \quad k<\hat{k} .
$$

Consequently, the system (3.1) at order $\hat{k}$ (written for the perturbed generalized equation) coincides with

$$
\left\{\begin{array}{l}
F_{z}^{\prime}\left(z^{0}, 0\right) z_{\hat{\xi}}^{\hat{k}}+\psi^{\dot{k}}\left(z^{0}, \ldots, z^{\dot{k}-1}\right)+\delta+\left(\begin{array}{c}
0 \\
\mu_{\hat{\delta}}^{k}
\end{array}\right)=0 . \\
\left(y_{\mathcal{\delta}}^{\dot{k}}\right)_{I}=0, \quad \mu_{\bar{I}}^{\hat{k}}=0,
\end{array}\right.
$$

where $\psi^{\dot{k}}$ is obtained as before from the expansion of $F\left(z_{\tilde{S}}^{\varepsilon}, \Xi\right)$ and $\bar{I}:=\{1, \ldots, p\}-I$. Choose $\delta=-\psi^{\hat{k}}\left(z^{0}, \ldots, z^{\hat{k}-1}\right)$. It follows from strong regularity that (3.11) has zero as unique solution which implies the uniqueness of the solution of the non homogeneous system. As a consequence, the Jacobian of (3.5) is non singular. We can thus apply to (3.5) the implicit function theorem and deduce the uniqueness of the Taylor expansion of (3.5), hence also the uniqueness of the solution of $(3.9)$.

ii) If the data are analytic, it follows from the implicit function theorem for real analytic functions(see e.g. Narasimhan [17] p. 17) that the solution of (3.5) is itself analytic.

\subsection{Linear equality constraints and finitly many linear inequality constraints}

We consider now the case when $E=Z^{\prime}$ and $N=N_{K}$, with

$$
\Lambda:=\{z \in Z ; A z=a, B z \leq b\}
$$

where $W$ is a Banach space, $A \in L(Z, W), B \in L\left(Z, \mathbb{E}^{p}\right),(a, b) \in \mathcal{W}:=W \times \mathbb{P}^{p}$. We assume that

$$
\left(\begin{array}{l}
A \\
B
\end{array}\right): Z \rightarrow \mathcal{W} \text { is onto, }
$$

which is equivalent (sec e.g. Brezis [2] p.29 Theorem II.19) to the coercivity of the adjoint (* denotes the adjoint operator) :

$$
\exists a>0 ; \forall(\lambda, \mu) \in \mathcal{W}^{\prime},\left\|A^{*} \lambda+B^{*} \mu\right\| \geq a(\|\lambda\|+\|\mu\|) .
$$

We define

$$
I(z):=\left\{i \in\{1, \ldots, p\} ;(B z),=b_{1}\right\} \text {. }
$$

We first recall a result on the structure of the normal cone (a straightforward consequence of Theorem 4, Chapter 1 of Ioffe-Tihomirov [s]) : 
Lemma 3.1 Let $K$ be defined by (3.12). Condition (3.13) implies that for any $z \in K$, the cone of outuard normals to $K$ at $z \in \Pi$ is

$$
N_{K}(z)=\left\{A \lambda+B \mu ; \lambda \in W^{\prime}, \mu \in \mathbb{R}^{p}, \mu \geq 0 ; \mu_{i}=0 \text { if } i \notin I(z)\right\} .
$$

Lemma 3.1 implies that in the polyhedral case, $(V I)_{\varepsilon}$ is equivalent to

$$
\left\{\begin{array}{l}
F(z, \varepsilon)+A^{*} \lambda+B^{*} \mu=0, \\
z \in K, \mu \geq 0,(B z-b)^{t} \mu=0 .
\end{array}\right.
$$

We have the following theorem as a consequence of Theorem 3.1.

Theorem 3.2 Assume that $z^{0}$ is a strongly regular solution of $(V I)_{0}$ with $N=N_{K}, K$ defined in (3.12), and denote by $\left(\lambda^{0} \cdot \mu^{0}\right)$ the associated multipliers. Assume that (3.13) holds. Then for all $k \geq 0$, there exists $\vec{\Sigma} \geq 0$ such that there exist $\tilde{\sim}^{1}, \ldots, z^{*}$ in $Z$ independent on $\varepsilon$ for $\varepsilon<\bar{\varepsilon}$ satisfying

$$
z^{*}=z^{0}+\varepsilon z^{1}+\cdots+\varepsilon^{k} z^{k}+O\left(\varepsilon^{k+1}\right),
$$

and a similar expansion holds for the multiplier, i.e.

$$
\mu^{\varepsilon}=\mu^{0}+\varepsilon \mu^{1}+\cdots+\varepsilon^{k} \mu^{k}+O\left(\varepsilon^{k+1}\right) .
$$

At each step $k \geq 1,\left(z^{k}, \mu^{k}\right)$ is the unique solution of

$$
\left\{\begin{array}{l}
F_{z}^{\prime}\left(z^{0}, 0\right) z^{k}+\psi^{k}\left(z^{0}, \ldots, z^{k-1}\right)+A^{*} \lambda^{k}+B^{*} \mu^{k}=0 \\
A z^{k}=0 \\
\left(B z^{k}\right)_{i}=0, i \in I_{+}^{k-1}, \\
\left(B z^{k}\right)_{i} \leq 0, \mu_{i}^{k} \geq 0, i \in I_{0}^{k-1}, \\
\mu_{i}^{k}=0, i \in I_{-}^{k-1}, \\
\left(\mu^{k}\right)^{t} B z^{k}=0 .
\end{array}\right.
$$

Proof It follows from the strong regularity of $z^{0}$ and (3.14) that, for $\varepsilon$ small enough, (3.15) has a unique solution $\left(z^{\varepsilon}, \lambda^{\varepsilon}, \mu^{\varepsilon}\right)$, where $z^{\varepsilon}$ is a solution of $(V I)_{\varepsilon}$.

The system (3.15) is equivalent to

$$
\left\{\begin{array}{l}
F(z, \varepsilon)+A^{*} \lambda+B^{*} \mu=0 \\
A z-a=0 \\
B z+y=b \\
y \geq 0, \mu \geq 0,\langle y, \mu\rangle=0
\end{array}\right.
$$


We may assume without loss of generality that $z^{0}=0$. hence $a=0$. Then $(3.17)$ can be rewritten as

$$
\mathcal{F}(z, y, \lambda, \mu . \Xi)+N(z, y, \lambda, \mu) \ni 0
$$

with

$$
\begin{gathered}
(z, y, \lambda, \mu) \in \mathcal{Z}:=\text { ker } A \times \mathbb{R}^{p} \times W^{\prime \prime} \times \mathbb{R}^{p}, \\
\mathcal{F}: \mathcal{Z} \times \mathbb{E}^{+} \rightarrow Z^{\prime} \times \mathbb{E}^{p} \times \mathbb{R}^{p}, \\
\mathcal{F}(z, y, \lambda, \mu, \Xi):=\left(\begin{array}{c}
F(z, \Xi)+A^{*} \lambda+B^{*} \mu \\
B z+y-b \\
-\mu
\end{array}\right) .
\end{gathered}
$$

and

$$
N(z, y, \lambda, \mu):=\left(\begin{array}{c}
\{0\}_{Z^{\prime}} \\
\{0\}_{\Sigma^{p}} \\
N_{\mathbb{R} p^{p}}(y)
\end{array}\right) .
$$

In order to apply Theorem 3.1, we only need to check that if $z^{0}$ is a SR solution of $(V I)_{0}$, then $\left(z^{0}, y^{0}, \lambda^{0}, \mu^{0}\right)$ is a SR solution of $(3.18)$ with $\Sigma=0$. Set

$$
z=z^{0}+\hat{z}, y=y^{0}+\hat{y}, \quad \lambda=\lambda^{0}+\hat{\lambda}, \quad \mu=\mu^{0}+\hat{\mu},
$$

and consider the linearized variational inequality:

$$
\left\{\begin{array}{l}
F\left(z^{0}\right)+F^{\prime}\left(z^{0}\right)\left(z-z^{0}\right)+A^{*}\left(\lambda^{0}+\hat{\lambda}\right)+B^{*}\left(\mu^{0}+\hat{\mu}\right)=\delta_{1}, \\
B z+y=b+\delta_{2} \\
-\mu^{0}-\hat{\mu}+N_{\bar{z} p+}^{r}\left(y^{0}\right)=\delta_{3} .
\end{array}\right.
$$

Because our analysis is local, we may suppose that $B z^{0}=b$, so that $y^{0}=0$. We set $\mu^{\sharp}=\hat{\mu}+\delta_{3}$. Using now (3.13) and that the second equality of (3.19) takes place in a finite dimensional space, we infer that $B$ has a linear right inverse, i.e. there exists $\hat{B} \in L\left(\mathbb{R}^{p}, \operatorname{ker} A\right)$ such that $\hat{B} \delta_{2}$ satisfies $B \hat{B} \delta_{2}=\delta_{2}$ (see [2], example 2 p.22 and Theorem II.10 p.23). Set $z^{\sharp}=\hat{z}-\hat{B} \delta_{2}$, system (3.19) is then equivalent to

$$
\left\{\begin{array}{l}
F\left(z^{0}\right)+F^{\prime}\left(z^{0}\right) z^{\sharp}+A^{*}\left(\lambda^{0}+\hat{\lambda}\right)+B^{*}\left(\mu^{0}+\mu^{*}\right)=\delta_{1}+B^{*} \delta_{2}-F^{\prime}\left(z^{0}\right) \hat{B}^{-1} \delta_{2}, \\
B z^{\sharp}+y \leq 0, \\
-\mu^{0}-\mu^{\sharp}+N_{\mathbb{R} p+}\left(y^{0}\right) \ni 0 .
\end{array}\right.
$$

which can be rewritten as

$$
F\left(z^{0}\right)+F^{\prime \prime}\left(z^{0}\right) z^{z}+\Lambda_{K}\left(z^{0}+z^{z}\right) \ni \delta_{1}+B^{*} \delta_{2}-F^{\prime}\left(z^{0}\right) \hat{B}^{-1} \delta_{2} .
$$

If the norm of $\delta=\left(\delta_{1}, \delta_{2}, \delta_{3}\right)$ is close enough to 0 . there exists a unique solution $z^{\sharp}(\delta)$ and for two given perturbations $(\delta, \eta)$. we have $\left|z^{*}(\delta)-z^{*}(\eta)\right|=O(\delta-\eta)$.

Coming back to $(\hat{z}, \hat{y}, \hat{\lambda}, \hat{\mu})$, we see that the same property holds for these variables. Strong regularity is thus proven. 


\section{Application to constrained optimization}

\subsection{Strong regularity and local optimality}

We consider a family of optimization problems

$$
\min f(x, \varepsilon) ; g(x, \varepsilon) \in \mathcal{K}
$$

where $X$ and $Y$ are Banach spaces, $\mathcal{K}$ is a closed convex cone in $Y^{\prime}$ and $f, g$ are $C^{\infty}$ mappings: $X \times \mathbb{R}^{+} \rightarrow \mathbb{R}, X \times \mathbb{R}^{+} \rightarrow Y^{\circ}$.

By $\nabla$ we denote the gradient operator with respect to $x$ only, and by $f(),. g($.$) we mean$ $f(., 0), g(., 0)$. To $(\mathcal{P})_{\varepsilon}$ is associated the optimality system

$$
\left\{\begin{array}{l}
\nabla f(x, \Xi)+g_{x}^{\prime}(x, \Xi)^{*} \lambda=0 \\
g(x, \Sigma) \in \mathcal{K}, \lambda \in \mathcal{K}^{-} \cdot\langle\lambda, g(x, \Sigma)\rangle=0
\end{array}\right.
$$

where $\mathcal{K}^{-}$is the polar cone defined by

$$
\mathcal{K}^{-}:=\left\{\mu \in Y^{-1}:\langle\mu, y\rangle \leq 0, \forall y \in \mathcal{K}\right\} .
$$

Set $Z:=X \times Y^{\prime \prime}$, and for $z:=(x, \lambda) \in Z$. define

$$
F(z . \Xi):=\left(\begin{array}{c}
\Gamma f(x, \Xi)+g_{x}^{\prime}(x, \Xi)^{*} \lambda \\
-g(x, \Xi)
\end{array}\right)
$$

and

$$
N(x, \lambda):=\{0\}_{X^{\prime \prime}} \times N_{-}(\lambda)
$$

with

$$
N_{-}(\lambda)= \begin{cases}\emptyset & \text { if } \lambda \notin \mathcal{K}^{-} \\ \left\{y \in \mathcal{K}^{-}:\langle y, \lambda\rangle=0\right\} & \text { if } \lambda \in \mathcal{K}^{-}\end{cases}
$$

We call standard form of the optimality system of $(\mathcal{P})_{\leq}$the following rariational inequality:

$$
F(z=)+V(z) \ni 0 \text {. }
$$

The following (classical) lemma is easily checked:

Lemma 4.1 A couple $z=(x, \lambda) \in \mathrm{X} \times \mathrm{I}$ is a solution of $(4.1)$ iff it is a solution of the standard form $(\mathcal{V I})_{s}$.

Remark 4.1 If $\mathrm{Y}$ is a refteriv Banach space. i.e. if the bidual $\mathrm{Y}^{-\prime}$ coincides with $Y$. then $N_{-}(\lambda)$ boils down to the cone of outuard normals to $\Lambda^{-}$at $\lambda$. In order to handle optimality systems in general Banach spaces. we have bern led to adopt a general format for $N$. 
At this point we may think of reducing the sensitivity analysis of local solutions of $(\mathcal{P})_{\varepsilon}$ to the analysis of solutions of $(\mathcal{V I})_{\varepsilon}$, thanks to the strong regularity condition. It remains however, to state the relations between the solutions of $(\mathcal{P})_{\varepsilon}$ and $(\mathcal{V I})_{\varepsilon}$. A first step is to check that SR implies the following qualification condition (see [14]).

Lemma 4.2 If $\left(x^{0}, \lambda^{0}\right)$ is a strongly regular solution of $(\mathcal{V I})_{0}$, then the qualification condition

$$
0 \in \operatorname{int}\left\{g\left(x^{0}\right)+g^{\prime}\left(x^{0}\right) X-\mathcal{K}\right\}
$$

holds.

Proof For any $\delta=(\theta, \eta)$ in $X^{\prime} \times Y$ of sufficiently small norm, the linearized generalized equation with r.h.s. $\delta$ admits a solution. Focusing our attention on the second term we find that

$$
-g\left(x^{0}\right)-g_{x}^{\prime}\left(x^{0}\right) d+N_{-}(\lambda+\mu) \ni \eta
$$

for some $(d, \mu) \in X \times Y^{\prime}$, and in particular

$$
g\left(x^{0}\right)+g_{x}^{\prime}\left(x^{0}\right) d-\mathcal{K} \ni-\eta
$$

As this is true for any $\eta$ such that $\|\eta\|$ is small enough, we recover (4.3).

The condition (4.3) implies the stability under perturbation of the set of feasible points, in the following sense:

Proposition 4.1 (Robinson [14], Theorem 1) If (4.3) holds, then there $\epsilon x i s t c>0, \varepsilon_{0}>0$ and a neighborhood $\mathcal{V}$ of $x^{0}$ such that

$$
\forall x \in \mathcal{V}, \varepsilon<\varepsilon_{0}, \exists \hat{x} ; g(\hat{x}, \varepsilon) \in \mathcal{K} ;\|x-\hat{x}\| \leq c \operatorname{dist}(g(x, \varepsilon), \mathcal{K}) .
$$

We now relate the SR condition and the quadratic growth condition (see e.g. Bonnans and Ioffe [3] for a discussion of this concept).

Definition 4.1 We say that $x^{0}$ satisfies the quadratic growth condition if there exist $\alpha>0$ and a neighborhood $\mathcal{V}$ of $x^{0}$ such that

$$
f(x) \geq f\left(x^{0}\right)+\alpha\left\|x-x^{0}\right\|^{2}, \forall x \in \mathcal{V} ; g(x) \in \mathcal{K}
$$

Let $T_{\mathcal{K}}(x)$ be the tangent cone to $\mathcal{K}$ at $x$. We know that (4.3) implies (see Kurcyusz [11]) that the closed convex set

$$
\mathrm{II}(x):=\left\{d \in \mathbb{X} ; g^{\prime}(x, \Xi) d \in T_{\mathcal{K}}[g(x, \Xi)]\right\}
$$

satisfies, for $=$ small enough and $x$ close to $x^{0}$ :

$$
N_{\Pi(x)}(0)=g^{\prime}(x, \Xi)^{*} N[g(x, \Xi)] .
$$


Theorem 4.1 Let $x^{0}$ be a local solution of $(\mathcal{F})_{0}$ and $\lambda^{0}$ its unique Lagrange multiplicr. If $\left(x^{0}, \lambda^{0}\right)$ is a strongly regular solution of $(\mathcal{V I})_{0}$, then

(i) the quadratic growth condition (4.4) holds for all $\alpha<1 /(\beta+1)^{2}$, where $\beta$ is the constant involved in Definition $\stackrel{0}{\sim} 1$.

(ii) there exists a neighborhood $\mathcal{V}$ of $\left(x^{0}, \lambda^{0}\right)$ such that for $\varepsilon$ small enough, $(\mathcal{P})_{\varepsilon}$ has a unique local solution $x^{\varepsilon}$, associated to a unique Lagrange multiplier $\lambda^{\varepsilon}$, such that $\left(x^{\varepsilon}, \lambda^{\varepsilon}\right)$ is the unique solution of $(\mathcal{V I})$ s in $\mathcal{V}$.

The proof is based on the following idea. Let $\left(x^{\varepsilon}, \lambda^{5}\right)$ be a solution of $(\mathcal{V I})_{\varepsilon}$. By $\gamma$-solution of an optimization problem, with $\gamma>0$, we mean a feasible point whose cost is lower or equal to $\gamma$ plus the infimum of the problem. Applying Ekeland's principle (stated below) to a $\gamma$-solution, we can associate another $\gamma$-solution, close to the first one, which minimizes an augmented cost function. Writing the associated optimality system and using SR we deduce that the new $\gamma$-solution cannot be far from $x^{5}$. This allows to check successively point (i) and (ii) of the theorem. We first recall Elieland's principle:

Proposition 4.2 (Ekeland [6]). Let $(E, d)$ be a complete metric space and $\varphi: E \rightarrow \mathbb{R} \cup\{+\infty\}$ a lower semi continuous (l.s.c.) function. Let $\varepsilon>0$ be given. If $\epsilon_{1} \in E$ is such that

$$
\varphi\left(\epsilon_{1}\right) \leq \inf \varphi+\varepsilon
$$

then there exists $e_{2} \in E$ such that $d\left(e_{1}, e_{2}\right) \leq \sqrt{\varepsilon}$ and

$$
\varphi\left(e_{2}\right) \leq \varphi(e)+\sqrt{\varepsilon} d\left(e, e_{2}\right), \forall e \in E .
$$

We derive from Ekeland's principle a lemma which is useful to prove Theorem 4.1. By $B$ we denote the unit ball of $X^{\prime}$.

Lemma 4.3 Let $\left(x^{0}, \lambda^{0}\right)$ satisfy the hypotheses of Theorem 4.1 and $x^{1}$ be a $\gamma$-solution of

$$
\min f(x, \varepsilon) ; g(x, \Xi) \in \mathcal{K} ;\left\|x-x^{0}\right\| \leq \theta,
$$

with

$$
\sqrt{\gamma} \leq 0 / 2 \text { and }\left\|x^{3}-x^{0}\right\|<\theta / 2 \text {. }
$$

If $\varepsilon>0$ is small enough, then there exists $\left(x^{2}, \lambda\right)$ in $X \times Y^{\prime}$ such that

$$
\begin{gathered}
\left\|x^{2}-x^{0}\right\|<\theta, \\
\left\|x^{2}-x^{1}\right\| \leq \sqrt{\gamma}, \\
\left\{\begin{array}{l}
\nabla f\left(x^{2}, \Xi\right)+g_{x^{\prime}}^{\prime}\left(x^{2}, \Xi\right)^{*} \lambda \in \sqrt{\gamma} B, \\
g\left(x^{2}, \varepsilon\right) \in \mathcal{K}, \lambda \in \mathcal{K}^{-},\left\langle\lambda, g\left(x^{2}, \varepsilon\right)\right\rangle=0 .
\end{array}\right.
\end{gathered}
$$


Proof Applying Proposition 4.2 to problem (4.6). with

$$
E:=\left\{x \in \mathbb{X}: g(x, \Xi) \in \mathcal{\Lambda}:\left\|x-x^{0}\right\| \leq \theta\right\}
$$

and

$$
\hat{\tau}(x):=f(x . \Sigma) \text {. }
$$

we infer the existence of $x^{2}$ satisfying $(4.9)$ and such that

$$
f\left(x^{2}, \Xi\right) \leq f(x . \Xi)+\sqrt{\gamma}\left\|x-r^{2}\right\| . \quad \forall x \in E .
$$

Relation (4.8) follows from (4.7) and (4.9). From (4.8) and (4.11) we deduce that $x^{2}$ is a local solution of

$$
\min _{x} f(x . \Xi)+\sqrt{i}\left\|x-x^{2}\right\|: g(x . \Xi) \in \mathcal{K} \text {. }
$$

Let $d \in X$ be such that $g^{\prime}\left(x^{2} . \Sigma\right) d \in T_{\mathcal{K}}\left[g\left(x^{2} . \Xi\right)\right]$. Proposition 4.1 implies that there exists a path $\mathbb{R}^{+} \rightarrow X, \sigma \rightarrow x(\sigma)=x^{2}+\sigma d+o(\sigma)$ and $g(x(\sigma) . \Xi) \in \mathcal{X}$. Hence, in direction $d$, the directional derivative of the cost cannot be negative : Consequently, $d=0$ is a solution of the convex problem

$$
\min _{d} f_{x}^{\prime}\left(x^{2}, \Xi\right) d+\sqrt{r_{i}}\|d\|: g_{x}^{\prime}\left(x^{2} . \Xi\right) d \in T_{\mathcal{K}}\left[g\left(x^{2} . \Xi\right)\right]
$$

Now (4.10) follows from subdifferential calculus and (-1.5).

\section{Proof of Theorem 4.1}

(i) Let $\theta>0$ be such that $x^{0}$ is a solution of

$$
\min \left\{f(x . \Sigma): g(x . \Sigma) \in \mathcal{K}:\left\|x-x^{0}\right\| \leq \theta\right\} .
$$

We consider a sequence $x^{k} \rightarrow x^{0}$. $x^{k} \neq x^{0}$. with $g\left(x^{k}, 0\right) \in \mathcal{K}$ and $f\left(x^{k}\right) \leq f\left(x^{0}\right)+$ $c\left\|x^{k}-x^{0}\right\|^{2}$ for some positive constant c. If no such sequence exists, this means that the quadratic growth condition is satisfied with an arbitrary value $\alpha$. Otherwise, we derive a positive under-estimate of $c$. Applying Lemma 4.3 with $\gamma=c\left\|x^{k}-x^{0}\right\|^{2}$ (hypothesis (4.7) is satisfied for $k$ large enough), we get the existence of $\left(\hat{x}^{k}, \lambda^{k}\right)$ in $X \times Y^{\prime}$ such that

$$
\begin{gathered}
\left\|\hat{x}^{k}-x^{k}\right\| \leq \sqrt{c}\left\|x^{k}-x^{0}\right\| . \\
\left\{\begin{array}{l}
\Gamma f\left(\hat{x}^{k}, 0\right)+g_{x}^{\prime}\left(\hat{x}^{k}\right)^{=} \lambda^{k} \in \sqrt{c}\left\|x^{k}-x^{0}\right\| B . \\
g\left(\hat{x}^{k}\right) \in \mathcal{K}, \lambda^{k} \in \mathcal{K}^{-},\left\langle g\left(\hat{x}^{k}\right), \lambda^{k}\right\rangle=0 .
\end{array}\right.
\end{gathered}
$$

Now by strong regularity and (4.1.3), as $\hat{x} \rightarrow x^{0}$, we have, for $k$ large enough

$$
\left\|\hat{x}^{k}-x^{0}\right\| \leq \beta \sqrt{c}\left\|x^{k}-x^{0}\right\|
$$


which implies with $(4.12)$ :

$$
\begin{aligned}
\beta \sqrt{c}\left\|x^{k}-x^{0}\right\| \geq\left\|\hat{x}^{k}-x^{0}\right\| & \geq\left\|x^{k}-x^{0}\right\|-\left\|x^{k}-\hat{x}^{k}\right\|, \\
& \geq(1-\sqrt{c})\left\|x^{k}-x^{0}\right\|,
\end{aligned}
$$

hence $(\beta+1) \sqrt{c} \geq 1$, i.e. $c \geq 1 /(\beta+1)^{2}$. Now choose $a<1 /(\beta+1)^{2}$. This means that there exists no sequence $x^{k} \rightarrow x^{0}, x^{k} \neq x^{0}$, such that $g\left(x^{k}\right) \in \mathcal{K}$ and $f\left(x^{k}\right) \leq f\left(x^{0}\right)+\alpha\left\|x^{k}-x^{0}\right\|^{2}$. From this, (4.4) follows for some neighborhood $\mathcal{V}$ of $x^{0}$.

(ii) Fix $\alpha<1 /(\beta+1)^{2}$. Let $\mathcal{V}$ be a neighborhood of $\left(x^{0}, \lambda^{0}\right)$ given by the strong regularity condition. Reducing if necessary the size of $\mathcal{V}$, we may assume that $\mathcal{V}=\mathcal{V}_{1} \times \mathcal{V}_{2}$ with $\mathcal{V}_{1} \in X$ and $\mathcal{V}_{2} \in I^{-1}$ and that the quadratic growth condition (4.4) is satisfied in $\mathcal{V}_{1}$. Let $\theta>0$ be such that $\mathcal{V}_{1} \subset B\left(x^{0}, \theta\right)$. We consider the set

$$
\text { - } s_{s}:=\left\{x \in \mathcal{I}: g(x, \Xi) \in \mathcal{K}: \frac{\theta}{2} \leq\left\|x-x^{0}\right\| \leq \theta\right\} \text {. }
$$

Using point (i) and Proposition 4.1. we infer that. if $\Xi$ and $\theta$ are small enough, we have

$$
x \in S_{s} \Rightarrow f(x) \geq f\left(x^{0}\right)+\frac{a \theta^{2}}{5} \text {. }
$$

Consider

$$
v_{\theta}(\Sigma):=\inf \left\{f(x . \Sigma): g(x . \Sigma) \in \mathcal{K}:\left\|x-x^{0}\right\| \leq \theta\right\} .
$$

Proposition 4.1 implies

$$
\exists x^{5} \in \mathcal{X} ; g\left(x^{2} . \Sigma\right) \in \mathcal{N}: x^{2}=x^{0}+O(\Sigma) .
$$

It follows that

$$
v_{\theta}(\xi) \leq f\left(x^{0}\right)+O(\equiv) \text {. }
$$

Now (4.14) and (4.16) imply that (for $z$ and $\theta$ small enough) there exists a minimizing sequence $\left\{x^{k}\right\}$ of $(4.15)$ which satisfies $\left\|x^{k}-x^{0}\right\| \leq 0 / 2$ for $k$ large enough. Denote $\gamma^{k}:=f\left(x^{k}, \varepsilon\right)-v_{\theta}(\varepsilon)$. Then $\gamma^{k} \rightarrow 0$. Using Lemma 4.3 we infer. for $\varepsilon$ and $\theta$ small enough, the existence of $\hat{x}^{k} \in \mathbb{X}^{*}$ and $\lambda^{k} \in I^{\prime}$ such that

$$
\left\{\begin{array}{c}
\left\|\hat{x}^{k}-x^{0}\right\| \leq \theta . \\
\nabla f\left(\hat{x}^{k} . \Xi\right)+g^{\prime}\left(\hat{x}^{k} . \Xi\right)^{*} \lambda^{k} \in \sqrt{\hat{i}^{k}} B . \\
g\left(\hat{x}^{k} . \Xi\right) \in \Lambda^{*} . \lambda \in \Lambda^{-} \cdot\left\langle\lambda . g\left(\hat{x}^{k} . \Xi\right)\right\rangle=0 .
\end{array}\right.
$$

Let $x^{\varepsilon}$ be the unique solution of $(\mathcal{L} \mathcal{I})$, in $\mathcal{L}$. Ising the strong regularity condition. we get $\left\|\hat{x}^{k}-x^{s}\right\| \leq \beta \sqrt{\gamma^{k}}$ : hence

$$
\left\|x^{k}-x^{\varepsilon}\right\| \leq\left\|x^{k}-\hat{x}^{k}\right\|+\left\|\hat{i}^{k}-x^{\varepsilon}\right\| \leq(3+1) \sqrt{\gamma^{k}}
$$

vanishes, i.e. $x^{k} \rightarrow x^{5}$ : it follows that $x^{5}$ is the unique local solution of $(\mathcal{P})_{\varepsilon}$ such that $\left\|x^{\varepsilon}-x^{0}\right\| \leq \theta$. 


\subsection{A sufficient condition for strong regularity}

We show here how some classical conditions involving qualification and positivity of the Hessian of the Lagrangian in some subspace of $X$ imply strong regularity. We define the Lagrangian

$$
L(x, \lambda, \Xi):=f(x, \varsigma)+\langle\lambda, g(x, \varepsilon)\rangle
$$

and state the following theorem related to Theorem 4.1 of Robinson [15] and the section 4 of Shapiro [18]. Note that a stronger result, involving more sophisticated conditions. has been obtained by $\mathrm{K}$. Malanowski in [12]. It is nevertheless worth presenting this result which can be proved in a simple way. By $L(x, \lambda)$ we denote $L(x, \lambda, 0)$.

Theorem 4.2 Let $x^{0}$ be a local solution of $(\mathcal{P})_{0}$ and $\lambda^{0}$ an associated Lagrange multiplier. We assume that

(i) $g^{\prime}\left(x^{0}\right)$ is onto,

(ii) $\exists \alpha>0 ;\left\langle d, \nabla^{2} L(x, \lambda) d\right\rangle \geq \alpha\|d\|^{2}, \forall d \in X, g^{\prime}\left(x^{0}\right) d \in \mathcal{K}-\mathcal{K}$.

Then $\left(x^{0}, \lambda^{0}\right)$ is a strongly regular solution of $(\mathcal{V} \mathcal{I})_{0}$.

Proof Consider the linearized generalized equation associated to $(\mathcal{V I})_{s}$, that is, denoting $\left(z-z^{0}\right)$ by $(d, \mu) \in X \times Y^{\prime}$ and $\delta=(\theta,-\eta)$ :

$$
\left\{\begin{array}{l}
\nabla f\left(x^{0}, 0\right)+g^{\prime}\left(x^{0}, 0\right)^{*} \lambda^{0}+\nabla^{2} L\left(x^{0}, \lambda^{0}\right) d+g^{\prime}\left(x^{0}, 0\right)^{*} \mu=\theta, \\
g\left(x^{0}\right)+g^{\prime}\left(x^{0}\right) d+N_{-}\left(\lambda^{0}+\mu\right) \ni \eta,
\end{array}\right.
$$

which appears to be the optimality system of

$$
\min _{d}\left\{f^{\prime}\left(x^{0}, 0\right) d-\langle\theta, d\rangle+\frac{1}{2}\left\langle d, \nabla^{2} L\left(x^{0}, \lambda^{0}\right) d\right\rangle\right\} ; g\left(x^{0}\right)+g^{\prime}\left(x^{0}\right) d-\eta \in \mathcal{K} .
$$

Using hypotheses (i) and (ii) of Theorem 4.2. it is easily checked that to each $(\theta, \eta)$ is associated a unique $(d, \mu)$ solution of $(4.18)$ and that $(4.17)$ has no other solution.

We establish now that if to $\delta^{i}=\left(0^{i} . \eta^{i}\right)$ is associated $\left(d^{i}, \mu^{i}\right), i=1.2$. solution of $(4.18)$ then

$$
\left\|d^{2}-d^{1}\right\|+\left\|\mu^{2}-\mu^{1}\right\|=O\left(\left\|\delta^{2}-\delta^{1}\right\|\right)
$$

Define

$$
l:=d^{2}-d^{1}, \mu:=\mu^{2}-\mu^{1}, \quad \theta:=\theta^{2}-\theta^{1}, \quad \eta:=\eta^{2}-\eta^{1},
$$

then

$$
\nabla^{2} L\left(x^{0} \cdot \lambda\right) d+g^{\prime}\left(x^{0}, 0\right)^{*} \mu=\theta,
$$

and consequently:

$$
\left\langle d, \nabla^{2} L\left(x^{0}, \lambda\right) d\right\rangle+\left\langle\mu, g^{\prime}\left(x^{0}, 0\right) d\right\rangle=\langle\theta, d\rangle .
$$


Moreover, we have for $i=1,2$ :

$$
g\left(x^{0}\right)+g^{\prime}\left(x^{0}\right) d^{i}-\eta^{i} \in \mathcal{K},
$$

and

$$
\eta^{i}-g\left(x^{0}\right)-g^{\prime}\left(x^{0}\right) d^{i} \in N\left(\lambda^{0}+\mu^{i}\right)
$$

which imply

$$
\begin{aligned}
& \left\langle\mu^{2}-\mu^{1} \cdot g\left(x^{0}\right)+g^{\prime}\left(x^{0}\right) d^{1}-\eta^{1}\right\rangle \leq 0 \\
& \left\langle\mu^{1}-\mu^{2} \cdot g\left(x^{0}\right)+g^{\prime}\left(x^{0}\right) d^{2}-\eta^{2}\right\rangle \leq 0
\end{aligned}
$$

therefore

$$
\left\langle\mu, g^{\prime}\left(x^{0}\right) d\right\rangle \geq\langle\mu, \eta\rangle .
$$

Together with $(4.20)$ we obtain

$$
\left\langle d, \nabla^{2} L\left(x^{0}, \lambda^{0}\right) d\right\rangle \leq\langle\theta, d\rangle-\langle\mu, \eta\rangle .
$$

Now by (4.21)

$$
g^{\prime}\left(x^{0}\right) d \in \mathcal{K}-\mathcal{K}+\eta .
$$

Since $g^{\prime}\left(x^{0}\right)$ is onto, there exists $c>0$ not depending on $(\eta, \theta)$ and $\hat{d}$ such that

$$
\begin{aligned}
& \|d-\hat{d}\| \leq c\|\eta\|, \\
& g^{\prime}\left(x^{0}\right) \hat{d} \in \mathcal{K}-\mathcal{K} .
\end{aligned}
$$

By $c_{1}, \ldots, c_{9}$ we denote positive constants non depending on $d$ or $\hat{d}$. We have

$$
\begin{aligned}
\left\langle d, \nabla^{2} L\left(x^{0}, \lambda^{0}\right) d\right\rangle & =\left\langle\hat{d}, \nabla^{2} L\left(x^{0}, \lambda^{0}\right) \hat{d}\right\rangle+2\left\langle d-\hat{d}, \nabla^{2} L\left(x^{0}, \lambda^{0}\right) \hat{d}\right\rangle \\
& +\left\langle d-\hat{d}, \nabla^{2} L\left(x^{0}, \lambda^{0}\right)(d-\hat{d})\right\rangle \\
& \geq \alpha\|\hat{d}\|^{2}-c_{1}\|\eta\|\|\hat{d}\|-c_{2}\|\eta\|^{2} .
\end{aligned}
$$

which leads, using (4.22) and (4.23), to

$$
\alpha\|\hat{d}\|^{2} \leq c_{1}\|\eta\|\|\hat{d}\|+c_{2}\|\eta\|^{2}+\|\theta\| .(\|\hat{d}\|+c\|\eta\|)+\|\mu\| .\|\eta\| .
$$

From (4.19), (4.2.3) and $g^{\prime}\left(x^{0}\right)$ onto, we have for some $c_{3}>0$ not depending on $d$ or $\hat{d}$

$$
\|\mu\| \leq c_{3}(\|d\|+\|\theta\|) \leq c_{3}(\|\hat{d}\|+c\|\eta\|+\|\theta\|) .
$$

With (4.24) we get an estimate of the type

$$
\|\hat{d}\|^{2} \leq c_{5}\|\hat{d}\|(\|\eta\|+\|\theta\|)+c_{6}(\|0\|+\|\eta\|)^{2} .
$$

Consequently, there exists $c_{-}>0$ such that

$$
\|\hat{d}\| \leq c_{-}(\|0\|+\|\eta\|)
$$

hence $\|d\| \leq\|\hat{d}\|+c\|\eta\| \leq c_{8}(\|\theta\|+\|\eta\|)$, and with (4.25) $\|\mu\| \leq c_{9}(\|\theta\|+\|\eta\|)$, as desired. 


\subsection{Computation and interpretation of the expansion}

We interpret here the expansion of the solution of constrained optimization problems $(\mathcal{P})_{\varepsilon}$. Expansion at order $k$ of $(\mathcal{V I})_{\varepsilon}$ is given by the general expression $(2.5)$ with $F=\left(\begin{array}{c}\nabla L \\ -g\end{array}\right)$ and $N$ defined in (4.2). The expansion $\Phi^{k}$ of $\left(\begin{array}{c}\nabla L \\ g\end{array}\right)$ up to order $k$ will be denoted by $\left(\begin{array}{c}\Phi_{\nabla L}^{k} \\ \Phi_{g}^{k}\end{array}\right)$ and similarly for $\psi^{k}=\left(\begin{array}{c}\psi^{k} \nabla L \\ \psi_{g}^{k}\end{array}\right)$ given by (2.4). We obtain

$$
\left\{\begin{aligned}
& \Phi_{\nabla L}^{k-1}\left(z^{0} \ldots, z^{k-1}(\varepsilon), \varepsilon\right)+\varepsilon^{k} \psi^{k} \cdot k \\
&+\varepsilon^{k}\left(\nabla^{2} L\left(z^{0}, \lambda^{0}\right) x^{k}(\varepsilon)+g_{x}^{\prime}\left(x^{0}\right)^{*} \lambda^{k}(\varepsilon)\right)=0, \\
&- {\left[\Phi_{g}^{k-1}\left(x^{0} \ldots \ldots x^{k-1}(\varepsilon)\right), \varepsilon\right)+\varepsilon^{k} \psi_{g}^{k}\left(x^{0} \ldots, x^{k-1}(\varepsilon)\right) } \\
&\left.+\varepsilon^{k} g_{x}^{\prime}\left(x^{0}\right) x^{k}(\varepsilon)\right] \in N_{-}\left(\lambda^{0}+\varepsilon \lambda^{1}(\varepsilon)+\cdots+\varepsilon^{k} \lambda^{k}(\varepsilon)\right) .
\end{aligned}\right.
$$

In order to write (4.26) as an optimality system with primal unknown $x^{k}$, we define

$$
\begin{gathered}
\lambda^{k}(\xi):=\lambda^{0}+\varepsilon^{1} \lambda^{1}(\xi)+\cdots+\varepsilon^{k} \lambda^{k}(\varepsilon), \\
c^{k}(\varepsilon):=\Phi_{\nabla L}^{k-1}\left(z^{0}, \ldots, z^{k-1}(\xi), \xi\right)+\varepsilon^{k} u_{\nabla L}^{k}\left(z^{0} \ldots, z^{k-1}(\varepsilon)\right)-g_{x}^{\prime}\left(x^{0}\right)^{*}\left(\lambda^{0}+\cdots+\varepsilon^{k-1} \lambda^{k-1}(\varepsilon)\right) .
\end{gathered}
$$

Then $(4.26)$ is equivalent to

$$
\left\{\begin{array}{l}
c^{k}(\varepsilon)+\varepsilon^{k} \nabla^{2} L\left(x^{0}, \lambda^{0}\right) x^{k}+g_{x}^{\prime}\left(x^{0}\right)^{*} \dot{\lambda}^{k}(\varepsilon)=0, \\
-\left[\Phi_{g}^{k-1}\left(x^{0}, \ldots, x^{k-1}(\varepsilon), \varepsilon\right)+\varepsilon^{k} \psi_{y}^{k}\left(x^{0}, \ldots, x^{k-1}(\varepsilon)\right)+\varepsilon^{k} g_{x}^{\prime}\left(x^{0}\right) x^{k}(\varepsilon)\right] \in N_{-}\left(\tilde{\lambda}^{k}(\varepsilon)\right)
\end{array}\right.
$$

which is the optimality system of

$$
\left\{\begin{array}{l}
\min _{d \in X}\left\{\left\langle c^{k}(\varepsilon), d\right\rangle+\frac{\xi^{k}}{2}\left\langle d, \nabla^{2} L\left(x^{0}, \lambda^{0}\right) d\right\rangle\right\}, \\
\Phi_{g}^{k-1}\left(x^{0}, \ldots, x^{k-1}(\varepsilon), \varepsilon\right)+\varepsilon^{k} u_{g}^{k}\left(x^{0} \ldots, x^{k-1}(\varepsilon)\right)+\varepsilon^{k} g_{2}^{\prime}\left(x^{0}\right) d \in \mathcal{K} ;
\end{array}\right.
$$

i.e., $x^{k}(\varepsilon)$ is obtained as the solution of an optimization problem with a quadratic cost and linear constraints, while $\tilde{\lambda}^{k}$ is the associated Lagrange multiplier.

\subsection{Problems with finitely many inequality constraints}

In this section we consider specialization of optimization problem $(\mathcal{P})_{\leq}$of the following type:

$$
\min f(x, \varepsilon) ; g_{1}(x, \varepsilon)=0 \text { in } Y_{1}, g_{2}(x, \varepsilon) \leq 0 \text { in }{ }^{p}
$$


so that

$$
\begin{aligned}
Y & =Y_{1} \times \Xi^{p} . \\
g(x, \Xi) & =\left(g_{1}(x, \Xi), g_{2}(x, \Xi)\right), \\
\mathcal{V} & =\{0\}_{\gamma_{1}} \times\{z \in \mathbb{E}, z \leq 0\},
\end{aligned}
$$

where $Y_{1}$ is a Banach space. 'Then. because the perturbation analysis is local, all the results remain true if we ignore the constraints that are not active at the point $x^{0}$. In this way, we deduce from Theorem 4.1 the following theorem (generalization of Theorem 4.1. of Robinson [15] to the case of an infinite number of equalities). We define

$$
I\left(x^{0}, 0\right)=\left\{i \in\{1, \ldots p\} \cdot g_{2 i}\left(x^{0}, 0\right)=0\right\}
$$

Theorem 4.3 Let $x^{0}$ be a local solution of $(\mathcal{P})_{0}$ and $\lambda^{0}$ an associated Lagrange multiplier. We assume that

(i) $\left(g_{1}^{\prime}\left(x^{0}\right),\left\{g_{2 i}^{\prime}\left(x^{0}\right)\right\}_{i \in I\left(x^{0} .0\right)}\right)$ is onto,

(ii) $\exists \alpha>0,\left\langle d, \nabla^{2} L\left(x^{0}, \lambda^{0}\right) d \geq a\|d\|^{2} . \forall d: g_{1}^{\prime}\left(x^{0}\right) d=0 . g_{2 i}^{\prime}\left(x^{0}\right) d\right.$ for $i$ s.t. $\lambda^{i}>0$,

then $\left(x^{0}, \lambda^{0}\right)$ is a strongly regular solution of $(\mathcal{V} \mathcal{I})_{0}$.

Let $\lambda=\left(\lambda_{1}, \lambda_{2}\right)$ be the multiplier associated to $g=\left(g_{1}, g_{2}\right)$. We observe that the associated generalized equation $(\mathcal{V I})_{\varepsilon}$ has data

$$
F(x, \lambda . \xi):=\left(\begin{array}{c}
\Gamma f(x . \xi)+\frac{g_{g}}{\partial x}(x . \Sigma)^{*} \lambda \\
-g_{1}(x . \Sigma) \\
-g_{2}(x, \Sigma)
\end{array}\right) .
$$

and

$$
X\left(x, \lambda_{1}, \lambda_{2}\right)=0_{x^{\prime}} \times 0_{\gamma_{1}} \times \lambda_{(\bar{z}+)^{p}}\left(\lambda_{2}\right) .
$$

We apply the results of section 3 with $z=\left(x, \lambda_{1}\right)$ and $y=\lambda_{2}$.

Using Remark 3.1, it is easy to check that the index sets $I_{+}^{p} . I_{0}^{p}$. $I_{-}^{p}$ have here the following interpretation:

$$
\begin{aligned}
I_{+}^{0} & =\left\{i \in\{1 \ldots p\}: \lambda_{i}^{0}>0\right\} . \\
I_{0}^{0} & =\left\{i \in\{1 \ldots p\}: g_{2 i}\left(x^{0}\right)=0=\lambda_{i}^{0}\right\}, \\
I_{-}^{0} & =\left\{i \in\{1 \ldots p\}: g_{2 i}\left(x^{0}\right)<0\right\} .
\end{aligned}
$$

and for $C \geq 1$ :

$$
\begin{aligned}
& I_{+}^{\ell}=I_{+}^{i-1} \cup\left\{i \in I_{0}^{t-1}: \lambda_{i}^{\ell}>0\right\} . \\
& I_{0}^{\dagger}=\left\{i \in I_{0}^{i-1}: \text { constraint } i \text { is active for }(S P)_{i} \text { and } \lambda_{i}^{\ell}=0\right\}, \\
& I_{-}^{\prime}=\left\{i \in I_{0}^{i-1}: \text { constraint } i \text { is not active for }(S P)_{i} \text { and } \lambda_{i}^{\ell}=0\right\},
\end{aligned}
$$


where $(S P)_{i}$ is defined as follows (compare to $(3.3$ and $(4.27))$ :

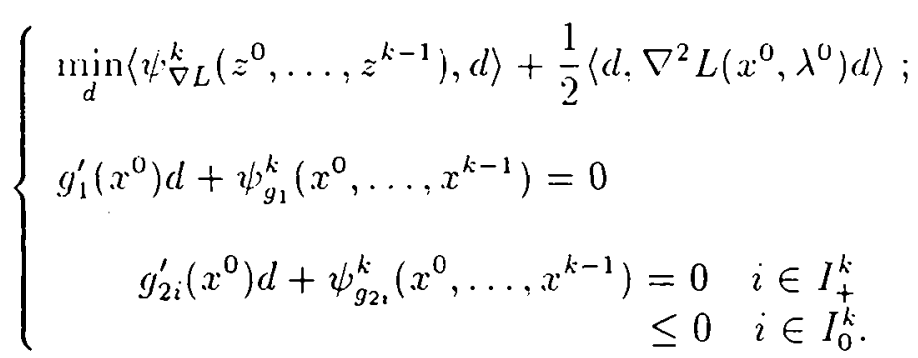

By Theorem 4.1, there exists for $\varepsilon$ small enough a unique couple $\left(x^{\varepsilon}, \lambda^{\varepsilon}\right)$ close to $\left(x^{0}, \lambda^{0}\right)$ such that $x^{\varepsilon}$ is the solution of (4.28) and $\lambda^{\varepsilon}$ is the unique associated Lagrange multiplier. Wie thus get the following result:

Theorem 4.4 Under the hypotheses of Theorem 4.3, the couple $\left(x^{\varepsilon} . \lambda^{s}\right)$ has a Taylor expansion. the term of order $k$ being defined by induction as the unique solution of (4.30).

We observed in section 3 that when all spaces are finite dimensional, if the data are analytic and if $I_{0}=\emptyset$, then $z^{\varepsilon}$ is itself analytic. However, assuming the strong second order condition (ii) of Theorem 4.3, we may remove the hypothesis on $I_{0}$.

Theorem 4.5 Assume that $X=\mathbb{R}^{n}, Y=\mathbb{R}^{q}$, the data are analytic with respect to $(z, \varepsilon)$ and the hypothesis of Theorem 4.3 hold. Then $\varepsilon \rightarrow\left(x^{\varepsilon}, \lambda^{\varepsilon}\right)$ is analytic.

Proof We apply Theorem 3.1 (ii). Let $I$ satisfy (3.4). It is sufficient to prove that the implicit function theorem can be applied to $(3.5)$; i.e. the corresponding homogeneous system

$$
\left\{\begin{array}{l}
F_{z}^{\prime}\left(z^{0}, 0\right) z+\left(\begin{array}{l}
0 \\
\mu
\end{array}\right)=0 \\
y_{i}=0, i \in I ; \mu_{i}=0, i \notin I
\end{array}\right.
$$

has only the trivial solution $(z, y, \mu)=(0,0,0)$. Coming back to the optimization problem (4.28), we see that the system $(4.31)$ is equivalent to

$$
\begin{gathered}
\nabla^{2} L\left(x^{0}, \lambda^{0}\right) d+g_{1}^{\prime}\left(x^{0}\right)^{*} \lambda+g_{2}^{\prime}\left(x^{0}\right)^{*} \mu=0 . \\
g_{1}^{\prime}\left(x^{0}\right) d=0, g_{2 i}^{\prime}\left(x^{0}\right) d=0 . i \in I ; \mu_{i}=0, i \notin I .
\end{gathered}
$$

Taking the scalar product of (4.32) with $x$ and taking into account (4.33) we obtain

$$
d^{t} \nabla^{2} L\left(x^{0}, \lambda^{0}\right) d=0
$$

which implies together with the second order condition (ii) of Theorem 4.3 that $d=0$. By condition (i) of Theorem 4.3, we deduce that $\lambda=0$ and $\mu=0$, as desired. 
It is interesting to compare Theorems 4.3 and 4.4 to the result of Jittorntrum [10]. Actually Jittorntrum proves that, if the gradients of active constraints are linearly independent and if the strong second order sufficient condition of Theorem 4.3 holds, then the solution of the optimization problem and the Lagrange multiplier have a directional derivative. Under the same assumptions, except for the $C^{\infty}$-differentiability of the data, we prove that the solution and the Lagrange multiplier have a power expansion at any order, and an expansion in powerseries if the data are analytic. In the case of convex problems, the high order expansion has already been obtained by Malanowski [13].

Acknowledgments : We thank G. Launay, K. Malanowski, A. Shapiro and S.M. Robinson for their useful remarks.

\section{References}

[1] Bensoussan, A. (198S). Perturbation methods in optimal control, Wiley, New York.

[2] Brezis, H. (1983). Analyse fonctionnelle: Théorie et applications, Masson, Paris.

[3] Bonnans, J.F.. Ioffe, A.D., (1993). Second order sufficiency and quadratic growth for non isolated minima. INRIA Report 1853.

[4] Bonnans, J.F.. Ioffe, A.D., Shapiro, A. (1992). Expansion of exact and approximate solutions in nonlinear programming. Advances in Optimization, W. Oettli and D. Pallaschke eds., Lectures Notes Econ. Math. Syst. 382, Springer Verlag, Berlin, 103-117.

[5] Bonnans, J.F., Shapiro, A. (1992). Sensitivity analysis of parametrized programs under cone constraints, SIAM J. Control Optimiz., 30, 1409-1422.

[6] Ekeland, I. (1979). Nonconvex minimization problems. Bull. Amer. Math. Soc. 1 (NS), $447-474$

[7] Ioffe, A.D. On sensitivity analysis of nonlinear programs in Banach spaces : the approach via composite unconstrained optimization, to appear.

[8] Ioffe, A.D., Tihomirov, V.M. (1974). Theory of extremal problems. Nauka, Moscow, (in russian). English translation : North Holland, 1979.

[9] Ito, K. , Kunish, K. (1992). Sensitivity analysis of solutions to optimization problems in Hilbert spaces with applications to optimal control and estimation. J. Diff. Equ. 99, 1-40

[10] Jittorntrum, K. (1984). Solution point differentiability without strict complementarity in nonlinear programming. Math. Prog. Study 21, 127-138.

[11] Kurcyusz, S. (1976). On the existence and nonexistence of Lagrange multipliers in Banach space. J. Optim. Theory Appl. 20, $81-110$. 
[12] Malanowski, K. (1992). Second-order conditions and constraint qualifications in stability and sensitivity analysis of solutions to optimization problems in Hilbert spaces. Appl. Math. Optim. 25, 51-79.

[13] Malanowski, K. (1987). Stability of Solutions to Convex Problems of Optimization. Lectures Notes in Contr. and Inf. Sci. 93. Springer Verlag. Berlin.

[14] Robinson, S.M. (1976). Stability theorems for systems of inequalities, Part II : differentiable nonlinear systems. SI.tM J. Nimer. Anal. 13. 497-513.

[15] Robinson, S.M. (1980). Strongly regular generalized equations. Math. Oper. Res. 5. 43-62.

[16] Robinson, S.M. (1991). An implicit-function theorem for a class of nonsmooth functions. Math. Oper. Res. 16. 29:2-309.

[17] Narasimhan, R. (1968). Analysis on real and Complex manifolds. Masson, Paris and NorthHolland, Amsterdam.

[18] Shapiro, A. Sensitivity analysis of parametrized programs via generalized equations. SIAM J. Control Optimiz.. to appear. 


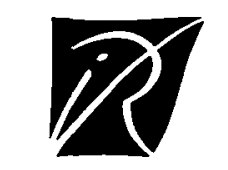

Unité de Recherche INRIA Rocquencourt Domaine de Voluceau - Rocquencourt - B.P. 105 - 78153 LE CHESNAY Cedex (France)

Unité de Recherche INRIA Lorraine Technopôle de Nancy-Brabois - Campus Scientifique 615, rue du Jardin Botanique - B P. 101 - 54602 VILLERS LES NANCY Cedex (France) Unité de Recherche INRIA Rennes IRISA. Campus Universitaire de Beaulieu 35042 RENNES Cedex (France)

Unité de Recherche INRIA Rhône-Alpes 46, avenue Félix Viallet-38031 GRENOBLE Cedex (France)

Unité de Recherchc INRIA Sophia Antipolis 2004, route des Lucioles - B.P. 93 - 06902 SOPHIA ANTIPOLIS Cedex (France)

EDITEUR

INRIA - Domaine de Voluceau - Rocquencourt - B.P. 105 - 78153 LE CHESNAY Cedex (France)

ISSN $0249-6399$ 